\title{
Complicaciones posquirúrgicas en pacientes sometidos a endarterectomía carotídea. Estudio transversal
}

\section{Post-surgical complications in patients undergoing carotid endarterectomy. Cross-sectional study}

\author{
Cristóbal Benítez-Rodríguez ${ }^{1}$, Miguel Á. Calderón-Llamas ${ }^{1}$, César Nuño-Escobar ${ }^{1}$, \\ Guadalupe Gavilánez-Chávez ${ }^{2,3}$, Francisco J. Llamas-Macías ${ }^{1}$, Jaime L. González-Bojórquez, \\ Jesús A. Guerrero-Jaureguit y Eva Ma. Olivas-Flores ${ }^{3,5 *}$
}

${ }^{1}$ Departamento de Angiología, Cirugía Vascular y Endovascular, Hospital de Especialidades Centro Médico Nacional de Occidente, Instituto Mexicano del Seguro Social; ${ }^{2}$ Servicio de Urgencias, Hospital General Regional $n .{ }^{\circ} 46$, Instituto Mexicano del Seguro Social; ${ }^{3}$ Programa de Doctorado en Farmacología, Centro Universitario de Ciencias de la Salud, Universidad de Guadalajara; ${ }^{4}$ Departamento de Anestesiología, Hospital General Regional n. ${ }^{\circ}$ 110, Instituto Mexicano del Seguro Social; ${ }^{5}$ Departamento de Anestesiología, Hospital de Especialidades Centro Médico Nacional de Occidente, Instituto Mexicano del Seguro Social. Guadalajara, Jalisco, México

\section{Resumen}

Antecedentes: La estenosis carotídea por ateroesclerosis es causante del 15-30\% de enfermedad vascular cerebral (EVC) de origen isquémico y en 2020 representó la séptima causa de muerte en México. La endarterectomía carotídea (ECA) es el tratamiento de elección para prevenir un EVC en pacientes con estenosis carotídea sintomática. Las complicaciones asociadas al tratamiento son infarto agudo al miocardio, hemorragia/hematomas, daño a nervios periféricos, EVC y muerte en el posquirúrgico inmediato. Objetivo: Determinar la prevalencia de las complicaciones posquirúrgicas en pacientes sometidos a endarterectomía por estenosis carotídea. Material y métodos: Estudio transversal analítico. Se analizaron pacientes como grupo 1 (con complicaciones, $n=6$ ), grupo 2 ( $\sin$ complicaciones, $n=16$ ). Se utilizó estadística descriptiva, comparativa e inferencial, se determinó un valor de $p<0.05$ como estadísticamente significativo. Se empleó el programa estadístico IBM SPSS V.28. Resultados: Se evaluaron 22 pacientes sometidos a ECA. La prevalencia de complicaciones fue del 27\%. La complicación más frecuente fue reintervención, en el 22.7\% ( $n=5)$, seguida de EVC 13.6\% ( $n=3)$, hematoma/hemorragia 9\% $(n=2)$ y secuela neurológica periférica 4.5\% $(n=1)$. Conclusiones: La necesidad de reintervención quirúrgica por hemorragia secundaria a la intervención y el EVC fueron las complicaciones mas frecuentemente encontradas (intervalo de confianza del 95\%: 0.59-0.99 [ $<$ 0.001] vs. 0.8-0.45 [ $p=0.023]$ ). Estos hallazgos serán útiles para posibles manejos perioperatorios en pacientes con estenosis carotídea.

Palabras clave: Endarterectomía carotídea. Ateroesclerosis. Evento vascular cerebral. Reintervención quirúrgica.

\section{Abstract}

Background: Carotid stenosis due to atherosclerosis is the cause of $15-30 \%$ of cerebral vascular disease (CVD) of ischemic origin and in 2020 it represented the seventh cause of death in Mexico. Carotid endarterectomy (CE) is the treatment of choice to prevent CVD in patients with symptomatic carotid stenosis. Complications associated with treatment are acute myocardial

Correspondencia:

${ }^{*}$ Eva Ma. Olivas-Flores

E-mail: eolivasflores@gmail.com

0377-4740/C 2021 Sociedad Mexicana de Angiología y Cirugía Vascular y Endovascular, A.C. PL bajo la licencia CC BY-NC-ND license (http://creativecommons.org/licenses/by-nc-nd/4.0/).
Disponible en internet: 29-11-2021 Rev Mex Angiol. 2021;49(4):117-122 www.RMAngiologia.com 
infarction, hemorrhage/bruising, peripheral nerve damage, CVD, and death in the immediate postoperative period. Objective: To determine the prevalence of postoperative complications in patients undergoing endarterectomy for carotid stenosis. Material and Methods: Analytical cross-sectional study. Patients were analyzed as group 1 (with complications $n=6$ ), group 2 (without complications $n=16$ ). Descriptive, comparative and inferential statistics were used, a value of $p<0.05$ was determined as statistically significant. Statistical program IBM SPSS V.28 was used. Results: 22 patients undergoing CE were evaluated. The prevalence of complications was $27 \%$. The most frequent complication was reoperation in $22.7 \%(n=5)$, CVD $13.6 \%(n=$ 3), hematoma/hemorrhage $9 \%(n=2)$, peripheral neurological sequelae $4.5 \%(n=1)$. Conclusions: The need for surgical reintervention due to bleeding secondary to the intervention and CVD were the most frequently found complications $195 \% \mathrm{Cl}$ $0.59-0.99, p<0.001$ vs. $0.8-0.45, p=0.023$ ). These findings will be useful for possible perioperative management in patients with carotid stenosis.

Key words: Carotid endarterectomy. Atherosclerosis. Cerebral vascular event. Surgical reintervention.

\section{Introducción}

La estenosis carotídea (EC) por ateroesclerosis es causante del $15-30 \%$ de enfermedad vascular cerebral (EVC) de origen isquémico ${ }^{1}$. En el 2020 representó la 7. ${ }^{\text {a }}$ causa de muerte en México².

La EC ateroesclerótica asintomática se refiere a la presencia de estrechamiento aterosclerótico de la arteria carótida interna extracraneal en individuos sin antecedentes de EVC isquémico del territorio carotídeo ipsilateral 0 ataque isquémico transitorio (AIT) $)^{3,4}$.

La prevalencia de EC ( $\geq 50 \%)$ en menores de 50 años fue de $0.2 \%$; para hombres y mujeres de 80 años o más fueron del 7.5 y $5 \%$, respectivamente. El riesgo estimado de EVC ipsilateral en pacientes con ECA asintomática (estenosis $\geq 50 \%$ ) es de aproximadamente $0.5-1.0 \%$, pero existe hasta un $5 \%$ de riesgo anual en $>80 \%$ de estenosis ${ }^{5}$.

Las pautas del Instituto Nacional para la Excelencia en la Salud y la Atención (NICE) y los criterios del Estudio Norteamericano de Estenosis Carotídea (NASCET) recomiendan manejo quirúrgico en el paciente que haya tenido un AIT o EVC con EC del $50-99 \%$ o EC del $70-99 \%$ con los criterios del estudio de la Sociedad Europea de Estenosis Carotídea (ESCT) debe de someterse a procedimiento quirúrgico en las siguientes dos semanas del evento ${ }^{4}$.

La endarterectomía carotídea (ECA) se ha considerado tradicionalmente como la técnica gold standard de tratamiento apoyado en los resultados de estudios aleatorizados históricos que demostraron su eficacia en prevención de eventos neurológicos frente al tratamiento médico aislado. Ha demostrado reducir el riesgo de presentar un accidente cerebrovascular ipsilateral en pacientes con EC sintomática, así como en pacientes seleccionados con EC severa asintomática ${ }^{6}$.

Los criterios para endarterectomía por EC sintomática de moderado a alto grado son: AIT ipsilateral o EVC isquémico no incapacitante, arteria carótida accesible quirúrgicamente, ausencia de enfermedades cardiacas, pulmonares o de otro tipo clínicamente significativas que aumenten en gran medida el riesgo de anestesia y cirugía, esperanza de vida de al menos cinco años, sin endarterectomía ipsilateral previa, riesgo de EVC perioperatorio y muerte para el cirujano o el centro debe ser $<6 \% 5,6$. Con un riesgo quirúrgico aceptable y una esperanza de vida de al menos cinco años, la ECA está claramente indicada para la estenosis sintomática del $50-70 \% 6,7$.

El informe del Stroke Council of American Heart Association y la Sociedad Europea de Cirugía Vascular (ESVS) reporta que las tasas de mortalidad y morbilidad neurológica aceptadas son un 3\% en pacientes asintomáticos, un $5 \%$ en pacientes con AIT y un $7 \%$ en pacientes con EVC previos ${ }^{8}$. En pacientes con EC bilateral, la lesión sintomática se aborda primero y el lado asintomático se aborda una vez que el paciente se ha recuperado de la primera ECA. Si ambos lados son asintomáticos y de gravedad similar, primero se trata la lesión del hemisferio dominante. Cuando una lesión es significativamente peor que la otra y ambas son asintomáticas, la lesión de mayor gravedad se aborda primero y la segunda después como un procedimiento por etapas ${ }^{9,10}$. La contraindicación absoluta de ECA es la oclusión carotídea completa asintomática. Contraindicaciones relativas: antecedentes de irradiación previa en el cuello, presencia de traqueotomía, disección radical del cuello previa con o sin radiación, parálisis de las cuerdas vocales contralaterales por EAC previa, ubicación de la lesión atípica alta o baja que es quirúrgicamente inaccesible, EC recurrente grave y riesgo médico inaceptablemente alto $0^{9,10}$.

LaECA realizadabajo anestesiageneral seasocióconel doble de probabilidades de sufrir infarto de miocardio intrahospitalario, cuatro veces más probabilidades de insuficiencia cardiaca congestiva aguda, 1.5 veces las 
probabilidades de inestabilidad hemodinámica y 1.8 veces las probabilidades de permanecer en el hospital durante $>1$ día $^{11,12}$. La incidencia de EVC en pacientes en los que se usó anestesia local fue del $3.2 \%$, en comparación con el 3.5\% en el grupo de anestesia general. Hubo una tendencia no significativa hacia una menor mortalidad operatoria con anestésico local. En el grupo de anestesia local, el $0.9 \%$ de los pacientes murieron dentro de los 30 días posteriores a la cirugía en comparación con el $1.5 \%$ de los pacientes del grupo de anestesia ${ }^{11}$.

No se ha encontrado que una técnica quirúrgica para la eliminación de placa sea superior a otra con respecto a la incidencia de EVC, muerte u otra morbilidad, la elección de la técnica depende en gran medida de las preferencias y la experiencia del cirujano ${ }^{9-11}$.

Con el uso de parche de vena o parche de politetrafluoroetileno(PTFE) parael cierre de ECA se ha observado que disminuye el riesgo de trombosis carotídea perioperatoria, EVC y reestenosis tardía del sitio de la endarterectomía ${ }^{11}$. Se ha reportado mayor incidencia de la dilatación aneurismática con el uso del parche de vena vs. el grupo de PTFE ${ }^{12}$.

El uso de shunt de modo sistemático podría ser innecesario en el $90 \%$ de los pacientes, se expone a riesgos de disección, embolia de placa durante la manipulación, embolia gaseosa, daño intimal que promueve trombosis postoperatoria temprana y reestenosis tardía ${ }^{13}$.

En cuanto a las complicaciones de ECA, en el ensayo International Carotid Stenting, la incidencia general de hematoma grave fue del $3.4 \%$ y en el estudio NASCET del $5.5 \%{ }^{14,15}$. El síndrome de hiperperfusión afecta a entre el 2 y $3 \%$ de los pacientes, suele alcanzar el máximo a los 2-7 días ${ }^{16}$.

Las lesiones de los nervios craneales (LNC) u otros nervios ocurren en aproximadamente el $5 \%$ de los pacientes después de unaECA. Las LNC se resuelven después de la cirugía y el riesgo de un déficit permanente de los pares craneales es bajo $(<1 \%)^{16}$.

En el estudio European Carotid Surgery Trial (ECST), la tasa de LNC motores en el periodo posoperatorio inmediato fue del $5.1 \%$, pero al momento del alta hospitalaria, la tasa de lesiones de los pares craneales se redujo al $3.7 \% \%^{17,18}$. El nervio hipogloso fue el más frecuentemente afectado, ocurriendo en un $2.7 \%$, seguido del nervio facial con un $1.9 \%$ y el nervio vago y el nervio glosofaríngeo cada uno con un $0.7 \%{ }^{19}$.

El objetivo de este estudio fue determinar la presencia de complicaciones posteriores a ECA por EC, en una unidad de tercer nivel de atención.

\section{Material y métodos}

Se realizó un estudio transversal retrospectivo. Se evaluaron los expedientes físicos y/o electrónicos de pacientes que fueron sometidos a ECA en el Hospital de Especialidades Centro Médico Nacional de Occidente en Guadalajara (Jalisco, México) del 1 de abril del 2017 al 31 de diciembre del 2020. Criterios de inclusión: pacientes mayores de 18 años, derechohabientes del Instituto Mexicano del Seguro Social (IMSS), diagnóstico de EC con base en ultrasonido Doppler, sometidosa ECA. Criterio de exclusión: no contar con expediente clínico completo. El estudio fue aprobado por el comité local de ética en investigación del Hospital de Especialidades de Centro Médico Nacional deOccidente en Guadalajara, Jalisco, México.

De acuerdo con la presencia o no de complicaciones se dividió a los pacientes en dos grupos, el grupo 1 (ECA con complicaciones, $n=6$ ) y el grupo 2 (ECA $\sin$ complicaciones, $n=16$ ). Se utilizó la prueba de Shapiro-Wilk para determinar la normalidad de la población. Para la descripción de las variables cualitativas se utilizaron frecuencias y porcentajes, para la descripción de variables cuantitativas se utilizaron medias y desviación estándar. Para las comparaciones entre variables cualitativas se utilizó la prueba exacta de Fisher, para las comparaciones entre variables cuantitativas la U de Mann-Whitney. Se realizó un análisis de regresión logística para determinar las variables asociadas a complicación de ECA; las covariables incluyen: edad y tiempo de pinzado. Se determinaron los intervalos de confianza del 95\% (IC 95\%). Se determinó un valor de $p<0.05$ como estadísticamente significativo. El análisis estadístico se realizó con IBM SPSS V.28.

\section{Resultados}

Se evaluaron 22 pacientes que fueron sometidos a endarterectomía por EC. La prevalencia de complicaciones posquirúrgicas fue del $27 \%$ (6 pacientes). Para el grupo 1 (ECA complicaciones, $n=6$ ) la media de edad fue de $73.5 \pm 8.36$, mientras que para el grupo 2 (ECA sin complicaciones, $n=16$ ) fue de $70 \pm 7.71$ ( $p=0.318)$. El resto de las características generales, datos demográficos y comorbilidades se encuentran en la tabla 1.

En cuanto a las características intraoperatorias para el grupo 1 y grupo 2, observamos riesgo según criterios de la Sociedad Americana de Anestesiología (ASA) similar, con $2.6 \pm 0.56$ y $3 \pm 0.59(p=0.252)$ respectivamente. Se sometió a anestesia local al $83 \%$ y al 
Tabla 1. Características generales de pacientes sometidos a endarterectomía carotídea $(\mathrm{n}=22)$

\begin{tabular}{|c|c|c|c|}
\hline Variable & Grupo $1(n=6)$ & Grupo 2 (n = 16) & $\mathbf{p}$ \\
\hline Edad, media $\pm \mathrm{DE}$ & $73.5 \pm 8.36$ & $70 \pm 7.71$ & $0.318^{*}$ \\
\hline $\begin{array}{l}\text { Sexo } \\
\text { Femenino, n (\%) } \\
\text { Masculino, n (\%) }\end{array}$ & $\begin{array}{l}4(66.7) \\
2(33.3)\end{array}$ & $\begin{array}{c}7(38.9) \\
11(61.1)\end{array}$ & $\begin{array}{l}0.235^{\dagger} \\
0.239^{\dagger}\end{array}$ \\
\hline $\begin{array}{l}\text { Hábitos } \\
\text { Tabaquismo, n (\%) }\end{array}$ & $4(66.7)$ & $15(83.3)$ & $0.862^{\dagger}$ \\
\hline $\begin{array}{l}\text { Comorbilidades } \\
\text { HAS, n (\%) } \\
\text { HAS, DM, n (\%) } \\
\text { DM2, n (\%) } \\
\text { HAS, HIPOT, ARRITMIA n (\%) } \\
\text { HAS, ARRITMIA, n (\%) } \\
\text { IAM, HAS, DM2, n (\%) } \\
\text { VC, AIT previo, n (\%) }\end{array}$ & $\begin{array}{c}1(16.7) \\
2(33.3) \\
-- \\
1(16.7) \\
-- \\
1(16.7) \\
4(66.7 \%)\end{array}$ & $\begin{array}{c}5(27.8) \\
8(44.4) \\
2(11.1 \%) \\
-- \\
1(5.6 \%) \\
14(77.8)\end{array}$ & $\begin{array}{c}0.366^{\dagger} \\
0.325^{\dagger} \\
- \\
- \\
0.768^{\dagger}\end{array}$ \\
\hline Cirugía carótida previa, n (\%) & $3(50 \%)$ & --- & - \\
\hline
\end{tabular}

$100 \%$ ( $p=0.655)$ respectivamente. El sangrado reportado en mililitros fue $90 \pm 84.14$ y $87.5 \pm 114.27$ ( $p=0.361)$, la prueba de pinzamiento fue de $4.67 \pm 1.03$ y $5.5 \pm 2.1$ ( $p=0.287)$; el tiempo de pinzamiento en minutos fue de $14.5 \pm 3.88$ y $15.5 \pm 5.33$ ( $p=0.406)$. Se realizó cierre primario en 5 (83.3\%) y 18 (100\%) $(p=0.278)$. El uso de shunt se presentó en 2 (33.3) y 2 (11.1) ( $p=0.076)$. En cuanto al tipo de drenaje, se usó Penrose en el $50 \%$ del grupo 1 y el $55.6 \%$ del grupo 2 ( $p=0.862)$, Drenovack en el 50 y $33.3 \%(p=0.335)$. Los días de estancia hospitalaria fueron $4.17 \pm 2.78$ para el grupo con complicaciones vs. $1.67 \pm 1.41$ para el grupo sin complicaciones ( $p=0.054$ ) (Tabla 2).

La prevalencia de complicaciones postoperatorias de ECA del total de pacientes en nuestro centro fue del $27.2 \%$, reintervención quirúrgica en el $22.7 \%$ (intervalo de confianza del 95\% [IC 95\%] de la odds ratio [OR]: 0.59-0.99, $p<0.01$ ), desarrollo de EVC del 13.6\% (IC 95\% OR: 0.8-0.45, $p=0.023)$, hematoma o sangrado 9\% (IC 95\% OR: 0.54-0.99, p = 0.84), muerte 9\% (IC 95\% OR:0.64-0-99, $p=0.088$ ) y desarrollo una secuela neurológica periférica 4.5\% (IC 95\% OR:) (Tabla 3).

\section{Discusión}

Diversos autores han descrito los beneficios de la ECA, sobre todo en pacientes con un porcentaje de oclusión mayor al $70 \%$ y la presencia de síntomas neurológicos (ataque isquémico transitorio [AIT], EVC previo al procedimiento $)^{6}$. Rerkasem, et al., en un metaanálisis observaron que los pacientes sometidos a ECA redujeron el riesgo a cinco años de cualquier accidente cerebrovascular o muerte operatoria (riesgo relativo [RR]: 0.77, IC 95\%: 0.63-0.94 y RR: 0.53, IC 95\%: 0.42-0.67) en pacientes con estenosis del 50 al $69 \%$ y estenosis del 70 al $99 \%$ respectivamente ${ }^{7}$.

La enfermedad carotídea por ateroesclerosis en México ocupa el 7.․ lugar de mortalidad según los datos del Instituto Nacional de Estadística y Geografía $\left(\right.$ INEGI) ${ }^{2}$. El objetivo de la ECA es disminuir el riesgo de EVC. Este procedimiento ha sido evaluado ampliamente en múltiples estudios prospectivos, sin embargo, no está exenta de presentar complicaciones. Según los estudios NASCET y ECST el EVC postoperatorio se observa en el 1 al $5 \%$ de los casos $^{4}$, en contraste con lo observado en nuestra población, donde el EVC postoperatorio se presentó en el $13.6 \%$ de los casos (IC 95\% OR: 0.8-0.45; p = 0.023). La incidencia de hematoma en el sitio de herida quirúrgica reportada por el estudio NASCET es de 5.4\% (RR 2.5, $p<0.001$ ); nuestro grupo de trabajo observó un $9 \%$, no se presentaron reportes de síndrome de hiperperfusión por esta causa. En cuanto a LNC, la literatura reporta un 5\%, observando en nuestro estudio un $4.5 \%$, similar a lo reportado. En cuanto a la tasa de mortalidad, la reportada en la literatura es del $3 \%$ de pacientes asintomáticos, el $5 \%$ con antecedente de AIT y el $7 \%$ en pacientes con antecedente de EVC previo; 
Tabla 2. Características intraoperatorias de pacientes sometidos a endarterectomía por estenosis carotídea (n = 22)

\begin{tabular}{|c|c|c|c|}
\hline Variable & Grupo $1(n=6)$ & Grupo $2(n=16)$ & $\mathbf{p}$ \\
\hline Riesgo ASA, media $\pm \mathrm{DE}$ & $2.6 \pm 0.56$ & $3 \pm 0.59$ & $0.252^{*}$ \\
\hline $\begin{array}{l}\text { Tipo de anestesia } \\
\text { Anestesia local, n (\%) } \\
\text { Anestesia general, n (\%) }\end{array}$ & $\begin{array}{l}5(83.3) \\
1(16.7)\end{array}$ & $\begin{array}{c}18(100) \\
---\end{array}$ & $0.655^{\dagger}$ \\
\hline Sangrado, media $\pm \mathrm{DE}$ & $90 \pm 84.14$ & $87.5 \pm 114.27$ & $0.361^{\dagger}$ \\
\hline Prueba de pinzamiento, media $\pm \mathrm{DE}$ & $4.67 \pm 1.03$ & $5.5 \pm 2.1$ & $0.287^{*}$ \\
\hline Tiempo de pinzamiento, media $\pm \mathrm{DE}$ & $14.5 \pm 3.88$ & $15.5 \pm 5.33$ & $0.406^{*}$ \\
\hline Cierre primario, media $\pm \mathrm{DE}$ & $5(83.3)$ & $18(100)$ & $0.278^{*}$ \\
\hline Cierre con parche, $\mathrm{n}(\%)$ & $1(16.7)$ & --- & \\
\hline Uso de shunt, $\mathrm{n}(\%)$ & $2(33.3)$ & $2(11.1)$ & $0.076^{\dagger}$ \\
\hline $\begin{array}{l}\text { Tipo de drenaje } \\
\text { Penrose, n (\%) } \\
\text { Drenovack, n (\%) }\end{array}$ & $\begin{array}{l}3(50) \\
3(50)\end{array}$ & $\begin{array}{c}10(55.6) \\
6(33.3)\end{array}$ & $\begin{array}{l}0.862^{\dagger} \\
0.335^{\dagger}\end{array}$ \\
\hline Estancia hospitalaria, media $\pm \mathrm{DE}$ & $4.17 \pm 2.78$ & $1.67 \pm 1.41$ & $0.054^{*}$ \\
\hline
\end{tabular}

Tabla 3. Factores asociados a complicaciones posquirúrgicas de pacientes sometidos a endarterectomía carotídea

\begin{tabular}{|c|c|c|c|c|}
\hline Variable & Casos $(n=6)$ & OR & IC 95\% & $\mathbf{p}$ \\
\hline Reintervención, n (\%) & $5(22.7)$ & & $0.59-0.99$ & $<0.001$ \\
\hline Evento vascular cerebral & $3(13.6)$ & & $0.8-0.45$ & 0.023 \\
\hline Hematoma/hemorragia & 2 (9) & & $0.54-0.99$ & 0.084 \\
\hline Muerte & 2 (9) & & $0.64-0.99$ & 0.088 \\
\hline Alteración neurológica periférica & $1(4.5)$ & & $0.60-0.96$ & 0.096 \\
\hline
\end{tabular}

OR: odds ratio; IC: intervalo de confianza.

nosotros observamos un $9 \%$ de mortalidad. Sin embargo, existen limitaciones en nuestro estudio: poco tamaño de la muestra y sesgo de información que tienen los estudios retrospectivos.

En su estudio del 2017, Vinogradov, et al. describen que la complicación de lesión de nervios periféricos son lesiones en su mayoría temporales, se ha descrito que ocurre entre el $2-17 \%$ de los pacientes, siendo la lesión del nervio hipogloso y del nervio laríngeo recurrente las más frecuentes, en nuestro grupo de pacientes con complicaciones postoperatorias tuvimos una lesión de nervio periférico manifestada por desviación de comisura labial en un paciente, por lo que se considera que nuestro centro se encuentra dentro del rango esperado para este tipo de lesiones (4.5\%).
La hemorragia de sitio quirúrgico o hematomas son una complicación posquirúrgica inmediata frecuente, se reporta en la literatura del 3-5.5\%; en nuestro centro se encontró del $9 \%$, la cual nos coloca en un muy elevado porcentaje de complicación por hematoma o sangrado, misma que requirió reexploración quirúrgica en la fase del posquirúrgico inmediato.

\section{Conclusiones}

La prevalencia de complicaciones posquirúrgicas en pacientes sometidos a ECA por EC es más frecuente que lo reportado en la literatura mundial (27.7\%). La elevada frecuencia en la necesidad de reintervenciones quirúrgicas por la presencia de hematoma o 
hemorragia incrementa el riesgo de EVC postoperatorio, por lo que es necesario hacer una evaluación preoperatoria integral que se base en la búsqueda de factores que incrementen el sangrado perioperatorio (uso de antiagregantes, anticoagulantes, etc.). No se realizó el uso de shunt de manera sistemática, fue utilizado solo en el $18 \%$ de los pacientes; a pesar de lo descrito en la literatura, donde apoyan el uso de cierre con parche, en nuestro centro solo se usó el parche en una ocasión $(3.5 \%)$ por decisión del cirujano, al igual que el tipo de técnica quirúrgica para endarterectomía convencional o tradicional. Sin duda estos hallazgos serán de utilidad para manejo preoperatorio y perioperatorio en pacientes para mejorar sus resultados postoperatorios con complicaciones menores.

\section{Agradecimientos}

Al Dr. Francisco Javier Llamas Macías, exjefe del Servicio de Angiología del Hospital de Especialidades Centro Médico Nacional de Occidente (CMNO). A las autoridades del Hospital de Especialidades CMNO, IMSS. A los médicos adscritos y personal que conforman el Servicio de Angiología y Cirugía Vascular y Endovascular.

\section{Financiamiento}

Los gastos generados en la elaboración del presente estudio estuvieron a cargo de los investigadores.

\section{Conflicto de intereses}

Los autores declaran que no existen conflictos de intereses.

\section{Responsabilidades éticas}

Protección de personas y animales. Los autores declaran que para esta investigación no se han realizado experimentos en seres humanos ni en animales.

Confidencialidad de los datos. Los autores declaran que han seguido los protocolos de nuestro centro de trabajo sobre la publicación de lo datos de los pacientes
Derecho a la privacidad y consentimiento informado. Los autores han obtenido el consentimiento informado de los pacientes y/o sujetos referidos en el articulo.

\section{Bibliografía}

1. Eliasziw M, Streifler JY, Fox AJ, Hacjinski VC, Ferguson GG, Barnett HJM. Significance of plaque ulceration in symptomatic patients with high grade stenosis. Stroke. 1994;25:3048.

2. Características de las defunciones registradas en México durante 2019 [Internet]. México: Instituto Nacional de Estadística y Geografía. Disponible en: www.inegi.org.mx

3. Mendes GA, Zabramski JM, Elhadi AM, Kalani MY, Preul MC, Nakaji P, et al. Carotid endarterectomy: comparison of complications between transverse and longitudinal incision. Neurosurgery. 2014;75:110-6.

4. Rocha JM, Pereira MJ, Dias MF, Andrade JP, Mansilha AA. Benefit of selective shunt use during carotid endarterectomy under regional anesthesia. Vascular. 2020;28(5):505-12.

5. Randomised trial of endarterectomy for recently symptomatic carotid stenosis: results of the MRC European Carotid Surgery Trial (ECST). Lancet. 1998;351(9113):1379-87.

6. Barnett HJ, Taylor DW, Eliasziw M, Fox AJ, Ferguson GG, Haynes RB, et al. Benefit of carotid endarterectomy in patients with symptomatic moderate or severe stenosis. North American Symptomatic Carotid Endarterectomy Trial Collaborators. N Engl J Med. 1998;339(20):1415-25.

7. Rerkasem A, Orrapin S, Howard DP, Rerkasem K. Carotid endarterectomy for symptomatic carotid stenosis. Cochrane Database Syst Rev. 2020;9:CD001081.

8. Yuan Y, Liao SY, Xin WQ. Carotid endarterectomy should be performed early or delayed in patients with symptomatic carotid stenosis? Clin Neurol Neurosurg. 2020;199:106317.

9. Knappich C, Kuehnl A, Haller B, Salvermoser M, Algra A, Becquemin JP, et al. Associations of perioperative variables with the 30-day risk of stroke or death in carotid endarterectomy for symptomatic carotid stenosis. Stroke. 2019;50:3439-48.

10. Vinogradov RA, Lashevich KA, Pykhteev VS. Comparing the risks for the development of perioperative complications in carotid endarterectomy and carotid angioplasty. Angiol Sosud Khir. 2017;23:133-9.

11. AbuRahma AF, Darling RC $3^{\text {rd }}$. Literature review of primary versus patching versus eversion as carotid endarterectomy closure. J Vasc Surg. 2021;74:666-75.

12. Orrapin S, Benyakorn T, Howard DP, Siribumrungwong B, Rerkasem K Patches of different types for carotid patch angioplasty. Cochrane Database Syst Rev. 2021;2:CD000071.

13. Joo SP, Cho YH, Lee YJ, Kim YS, Kim TS. Modified suturing techniques in carotid endarterectomy for reducing the cerebral ischemic time. J Korean Neurosurg Soc. 2020;63:834-40.

14. Martín-Morales $E$, Jiménez-Román $R$, Paluso-Montero A, Hernández-Ruiz $T$, Mendieta-Azcona C, Moral LFR. Results and complications of carotid endarterectomy in a hospital from Madrid, Spain. Cir anesthesia. 2019;87:501-7.

15. Müller MD, Lyrer $\mathrm{P}$, Brown MM, Bonati LH. Carotid artery stenting versus endarterectomy for treatment of carotid artery stenosis. Cochrane Database Syst Rev. 2020;2:CD000515.

16. Doig D, Turner EL, Dobson J, Featherstone RL, de Borst GJ, Brown MM, et al.; ICSS Investigators. Incidence, impact, and predictors of cranial nerve palsy and haematoma following carotid endarterectomy in the international carotid stenting study. Eur J Vasc Endovasc Surg. 2014;48:498-504.

17. Barnett HJ, Taylor DW, Eliasziw M, Fox AJ, Ferguson GG, Haynes RB, et al. Benefit of carotid endarterectomy in patients with symptomatic moderate or severe stenosis. N Engl J Med. 1998;339:1415.

18. Roseborough GS, Perler BA. Carotid Artery Disease: Endarterectomy. En: Rutherford's vascular Surgery. $7^{\text {th }}$ Ed. Philadelphia: Elsevier; 2010. p. 2090

19. Fokkema M, de Borst GJ, Nolan BW, Indes J, Buck DB, Lo RC, et al.; Vascular Study Group of New England. Clinical relevance of cranial nerve injury following carotid endarterectomy. Eur J Vasc Endovasc Surg. 2014;47:2-7 